\title{
THE METHOLOGY FOR DETERMINATION OF CATALYTIC PROPERTIES OF SiC SAMPLES FOR SPECIFIED PRE-X AND MSRO CONDITIONS
}

\author{
V.I. Sakharov ${ }^{1}$, A. F. Kolesnikov ${ }^{2}$, A. N. Gordeev², \\ and J.-L. Vérant ${ }^{3}$ \\ ${ }^{1}$ Institute of Mechanics \\ M. V. Lomonosov Moscow State University \\ Michurinskii Prosp. 1, Moscow 119192, Russia \\ ${ }^{2}$ Institute for Problems in Mechanics \\ Russian Academy of Sciences \\ Prosp. Vernadskogo 101/1, Moscow 119526, Russia \\ ${ }^{3}$ ONERA DMAE/C2A
} Av. E. Belin 2, Toulouse 31055, France

\begin{abstract}
Numerical simulation of subsonic inductively coupled plasma (ICP) flow and exhausted air and $\mathrm{CO}_{2}$-plasma jets around of the flat-face model with $\mathrm{SiC}$ coating were performed for IPG-4 (IPM RAS) facility test regimes simulating of reentry heating of the Pre-X space vehicle (Earth) and the MSRO - Mars Sample Return Orbiter (MSRO) (Mars). The calculation of the body heat flux maps was based on numerical solution of one-dimensional equations of laminar thermally and chemically nonequilibrium multicomponent boundary layer with finite thickness. The necessary flow data at the outer edge of the boundary layer were taken from results of Navier-Stokes calculations. The result obtained in the framework of macroscopic approach shows that the $\mathrm{SiC}$ samples are a low catalytic material in regards with the specified operating conditions for the Pre-X and for the MSRO.
\end{abstract}

\section{INTRODUCTION}

The INTAS/CNES project 53-5117 has been generated by the interest of CNES and ONERA in two types of projects involving high-speed atmospheric flight: Mars exploration with Mars atmosphere entry vehicles and the preliminary stud-

This is an Open Access article distributed under the terms of the Creative Commons Attribution-Noncommercial License 3.0, which permits unrestricted use, distribution, and reproduction in any noncommercial medium, provided the original work is properly cited. 


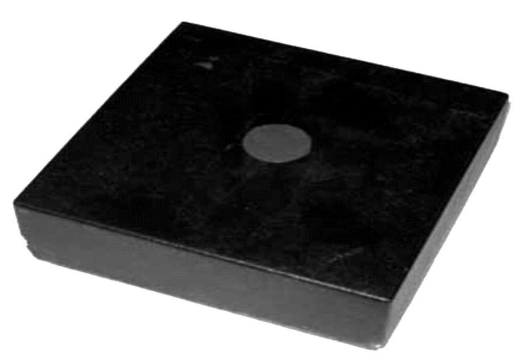

(a)

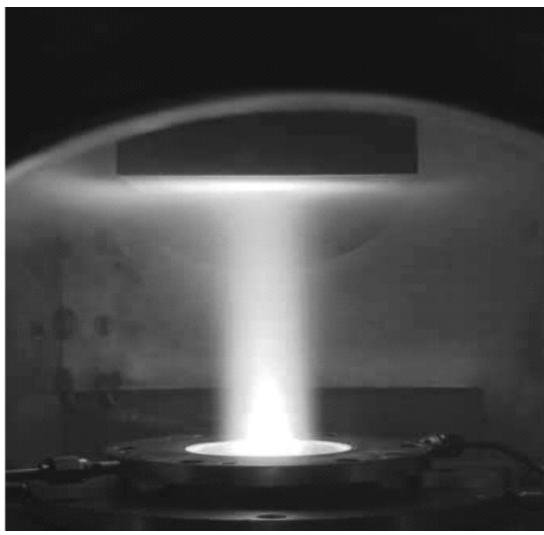

(b)

Figure $1 \mathrm{SiC}$ sample and its ICP air test (IPM IPG-4 plasmatron)

ies for future reusable launchers. In both cases, a correct evaluation of material catalysis properties of the Thermal Protection System (TPS) represents an important issue.

The goals of the INTAS/CNES project 53-5117 was to demonstrate a methodology based on macroscopic and microscopic approaches to describe catalytic processes as a major element for improvement of TPS design in the way of increasing safety and heating margin diminishing [1]. Moreover, increasing the knowledge of catalytic processes with updated results or models allows validating the chain ground experiments - numerical codes. The measurement techniques, Computational Fluid Dynamics (CFD) modeling, provide the basis for complete local duplication of stagnation point heat fluxes to a vehicle surface at trajectory points which should be selected. This multidisciplinary approach reveals the way for aerothermal testing Thermal Protecting Materials (TPM) samples and determination of TPM catalysis for entry conditions and to construction the bridge from plasmatron test to hypersonic flight through the Martian and Earth atmospheres. Finally, the TPM catalytic efficiencies extracted from the data of heat transfer rates will be used for developing models of surface catalysis and prediction of the vehicle surface heating during atmospheric entry.

In order to achieve such goals, the $\mathrm{SiC}$ samples (provided by CNRS) to be tested using facility (Fig. 1) in Institute for Problems in Mechanics of the Russian Academy of Sciences (IPM RAS) and, moreover, experimental tests requested to be performed on the same specified Pre-X RLV (Reusable Launch Vehicle) conditions (Earth) and MSRO conditions (Mars).

The IPM facility is the inductively heated plasma wind tunnel: 100-kilowatt IPG-4 plasmatron. The Inductively Coupled Plasma (ICP) torch guarantees the 
purity of the plasma flow and consequently allows determining surface catalysis properties more accurately than in any classical arc jets.

In IPM, a few TPM, including silica-based tile surface, have been examined and surface catalytic efficiencies were determined at high temperatures and pressure around $10000 \mathrm{~Pa}$.

The theoretical background of the scaling stagnation point heating is based on the concept of the local simulation of stagnation point heat transfer, also known as LHTS, which has been developed by IPM $[2,3]$. Also, the methodology of LHTS for the Earth and Martian atmospheres entry conditions has been adapted for the IPG-4 facility [4].

This concept requires that the three key parameters - total enthalpy, stagnation pressure, and velocity gradient - which control the stagnation point heat transfer, have to be equal in hypersonic flight and in ground test. If the flows in the ICP facility and at the free flight conditions are under local thermochemical equilibrium outside of the boundary layer, these requirements provide the local similarity of the reacting boundary layer and heat transfer rate at the stagnation point. This concept has been validated for high enthalpy subsonic flows of nitrogen, $\mathrm{CO}_{2}$, and air [5].

The methodology and technical approach for these studies were based on combined measurements and CFD modeling of plasma reacting gas flows and heat transfer for the plasmatron test conditions.

The experimental characterization has included measurements of the plasma temperature, dynamic pressure, stagnation point heat flux and pressure, TPM surface temperature in subsonic carbon dioxide flows and subsonic air flows.

An indispensable part of the IPM methodology for rebuilding plasma free stream conditions and TPM catalysis determination is CFD modeling of plasma flows and heat transfer for test conditions [6].

The CFD tools developed in IPM are already included in the menu of the IPG-4 plasmatron instruments and characterization techniques for air and $\mathrm{CO}_{2}-$ plasma tests. In the framework of INTAS/CNES project, progress of Institute of Mechanics, Moscow State University (IM MSU), has been adapted on the entire facility operating envelope with Navier-Stokes (N-S) approach. For this purpose, the model of plasma flows under thermal and chemical nonequilibrium has been developed and implemented into CFD codes along with developing and validating advanced codes and computational technologies.

\section{NUMERICAL MODELING}

The numerical methodology developed by IPM is robust and gives good results for catalycity and heat flux predictions. According to IPM approach, the whole region of the flow in the plasmatron is subdivided into three regions: $(i)$ induc- 
tively coupled plasma flow in the torch; $(i i)$ jet flow around the model in the test chamber; and (iii) boundary layer in front of the model stagnation point.

Each region is computed separately.

The main aim of CFD modeling of the flow in the first two regions under the chemical equilibrium assumption is to determine the set of dimensionless parameters at the outer edge of boundary layer in front of the model stagnation point. These dimensionless parameters will be used in further boundary layer computations in order to calculate theoretical heat flux in the model stagnation point.

The IM MSU approach has been developed for simulation this problem without dividing a domain into different subregions. The chemically and chemically nonequilibrium flows in a discharge channel of the inductive plasmatron IPG-4 and in underexpanded supersonic air jets around a flat-end model were studied by CFD modeling [7-9].

The methodology employed by IM MSU for modeling supersonic issues dealing with nonequilibrium (chemically and thermally) flow has been adapted for subsonic ones by using low Mach preconditioning techniques. The aimed goal was to take benefit of IM MSU ability for physical and numerical developments to improve the methodology used by IPM, either to confirm or not the equilibrium assumption. In this latter case, accounting for the nonequilibrium influence will extend the use of the IPM methodology.

The air in ICP flow is considered as a mixture of 11 species: $\mathrm{O}, \mathrm{N}, \mathrm{O}_{2}$, $\mathrm{N}_{2}, \mathrm{NO}, \mathrm{O}^{+}, \mathrm{N}^{+}, \mathrm{O}_{2}^{+}, \mathrm{N}_{2}^{+}, \mathrm{NO}^{+}$, and $e$. The chemical model for gas phase reaction in the $\mathrm{CO}_{2}$ case consists also of a 11 species mixture: $\mathrm{O}, \mathrm{C}, \mathrm{O}_{2}, \mathrm{CO}$, $\mathrm{C}_{2}, \mathrm{CO}_{2}, \mathrm{O}^{+}, \mathrm{C}^{+}, \mathrm{CO}^{+}, \mathrm{O}_{2}^{+}$, and $e$. Rotational and vibrational energy modes of molecules are described by the "rigid rotator-harmonic oscillator" model with the Boltzmann distribution of the energy levels. It is supposed that all species are in the ground electronic state and rotational temperature of molecules is equal to translational temperature $T$. Three nonequilibrium thermochemical models have been developed to be used in CFD modeling:

- one-temperature model where vibrational and electron temperatures are assumed to be equal to translational temperature $T$;

- two-temperature model with relaxation of the vibrational energy modes of molecules $\mathrm{O}_{2}, \mathrm{~N}_{2}$, and $\mathrm{CO}_{2}$ characterized by common vibrational temperature $T_{v}$ differed from translational temperature $T$. The $V T$ and $e V$ energy exchanges were considered. The temperature of electrons was assumed to be equal to vibrational temperature; and

- three-temperature model with common vibrational temperature $T_{v}$ for molecules $\mathrm{O}_{2}, \mathrm{~N}_{2}$, and $\mathrm{CO}_{2}$ differed from translational temperature $T$ and electron temperature $T_{e}$ calculated from the electron gas energy balance 
equation. The $V T, e T$, and $e V$ energy exchanges were taken into account in the numerical modeling.

In order to describe viscous fluxes of mass, momentum, and energy in gas, special attention must be given to the transport properties. The molar diffusion fluxes are determined from the two-temperature Stefan-Maxwell relations for the partially ionized gas mixture including temperatures of electrons and heavy particles [10]. The collision integrals of diffusion and viscous types are calculated using approximate formulas obtained by evaluation from potentials for various pairs of species [11]. The viscosity and thermal conductivity of air mixture are calculated by approximate Wilke-Vasiliyeva formulas [12].

This problem has been solving in on the basis of effective of $\mathrm{N}-\mathrm{S}$ numerical technique for high temperature gas flow simulation (HIGHTEMP) [13]. The quasi-three-dimensional time-dependent $\mathrm{N}-\mathrm{S}$ equations are integrated on the multiblock structured mesh using a finite volume approach. The steady-state solution is defined due to implicit two-layer iterative procedure. Implicit linear operator is similar to quasi-linear form of governing equations. Operator is solved by the Gauss-Seidel line space-marching method with LU-decomposition of the block-tridiagonal matrices to find solution in every line step.

Considering the plasma generation, this problem has been solving on the basis of $\mathrm{N}-\mathrm{S}$ equations coupled with the simplified Maxwell equation for the RF (radio frequency) electromagnetic field. Simplified quasi-one-dimensional Maxwell equations for the complex amplitude of the electric field tangential component generating circular electric currents were solved for the discharge channel [6].

The calculation of heat transfer and catalycity at the stagnation point is based on numerical solution of one-dimensional equations of laminar nonequilibrium multicomponents boundary layer (BL) with finite thickness with account for flow vorticity at the outer edge of the layer.

It is assumed the gas is represented by a five-species (for air) and by a sixspecies (for $\mathrm{CO}_{2}$ ) neutral mixtures. The flow at the outer edge of $\mathrm{BL}$ and within the BL is supposed to be in chemical nonequilibrium and (or not) in thermal nonequilibrium. The diffusion processes are described by multicomponent diffusion law. The probabilities of surface recombination of particles are assumed to be equal; so, the single parameter - effective surface recombination probability $\gamma$ can be determined.

\section{$3 \quad$ PRE-X DUPLICATED AIR CONDITIONS}

The three trajectory points of the Pre-X vehicle (equivalent nose radius $R_{n}$ is $925 \mathrm{~mm}$ ) were chosen in order to simulate the stagnation point heating in the IPG-4 plasmatron. The flight conditions at the three trajectory points are 
Table 1 Flight conditions at three trajectory points

\begin{tabular}{ccccc}
\hline Trajectory point, & $V_{\infty}, \mathrm{m} / \mathrm{s}$ & Heat flux, $\mathrm{kW} / \mathrm{m}^{2}$ & $T_{\mathrm{re}}, \mathrm{K}$ & $P_{\text {st }}, \mathrm{Pa}$ \\
\hline 1 & 7230 & 540 & 1830 & 2000 \\
2 & 6657 & 584 & 1866 & 3748 \\
3 & 5584 & 528 & 1819 & 7607 \\
\hline
\end{tabular}

Table 2 Parameters of the IPG-4 testing regimes for fully catalytic conditions and radiative equilibrium wall temperature

\begin{tabular}{cccccc}
\hline Regime IPG-4, air & $\begin{array}{c}N_{\mathrm{ap}}, \\
\mathrm{kW}\end{array}$ & Efficiency & $\begin{array}{c}\text { Heat flux, } \\
\mathrm{kW} / \mathrm{m}^{2}\end{array}$ & $\begin{array}{c}P_{\mathrm{ch}}, \\
\mathrm{Pa}\end{array}$ & $\begin{array}{c}Z_{\text {exit }}, \\
\mathrm{mm}\end{array}$ \\
\hline 1 & 45.7 & 0.35 & 550 & 2000 & 130 \\
2 & 52.0 & 0.375 & 580 & 3800 & 130 \\
3 & 29.4 & 0.415 & 530 & 7800 & 100 \\
\hline
\end{tabular}

given in Table 1 . Here, $V_{\infty}$ is the reentry velocity; heat flux is the value to fully catalytic radiative equilibrium wall; $T_{\text {re }}$ is the radiative equilibrium wall temperature; and $P_{\text {st }}$ is the stagnation pressure.

Preliminary analysis based on LHTS theory showed and high-enthalpy subsonic tests in IPG-4 plasmatron confirmed that the specified stagnation point of heat fluxes to fully catalytic wall can be realized using the water-cooled flat-faced 140-millimeter diameter cylindrical model made of copper, which have been used to study heat transfer at IPM. This model was refined to use it not only for heat flux measurements, but for dynamic pressure measurements as well. The experimental data for the three IPG-4 operating regimes corresponding to the Pre-X trajectory are provided in Table 2 .

The new testing model was developed and manufactured in IPM on the basis of full scale $(150 \times 150 \times 45 \mathrm{~mm})$ light-weight silica-based thermal protection tile with $\mathrm{SiC}$ sample installed in it.

\subsection{Plasma Air Flow Simulation}

The test numerical calculations were performed for simplified modeling of subsonic airflow over infinity plane as well as around the cylindrical model with diameter $D=140 \mathrm{~mm}$ located in the test chamber. In general, the geometry of the subsonic air jets was found to be in a good agreement with experimental results for both approaches. The stagnation point heat fluxes for the flows over cupreous cylindrical model vs. the flow over infinity plane were identical and close to test ones. The further numerical simulations have been carried out for 


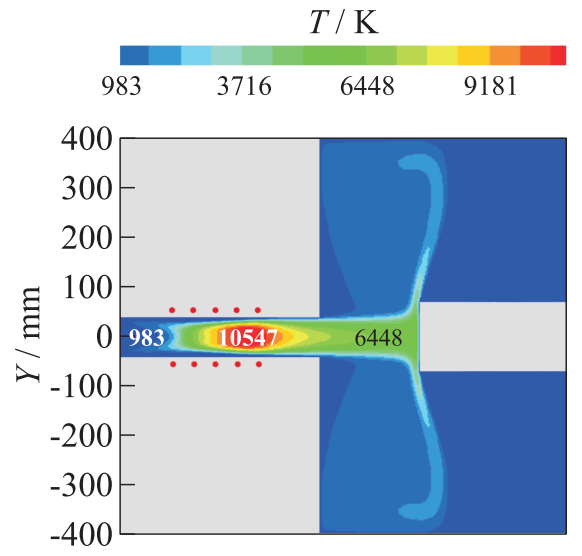

(a)

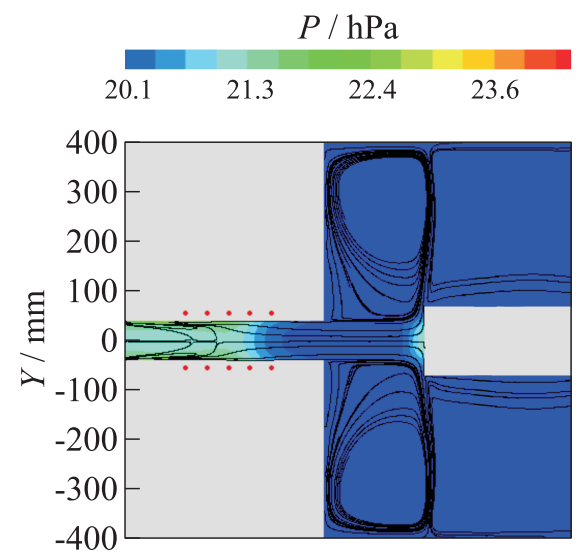

(b)

Figure 2 The temperature $(a)$ and pressure $(b)$ contours and streamlines: air, regime \#1 ( $P=2000 \mathrm{~Pa})$. (Refer Sakharov et al., p. 371.)

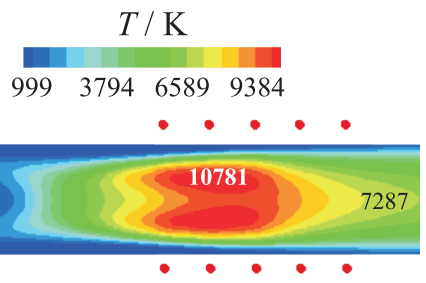

(a)

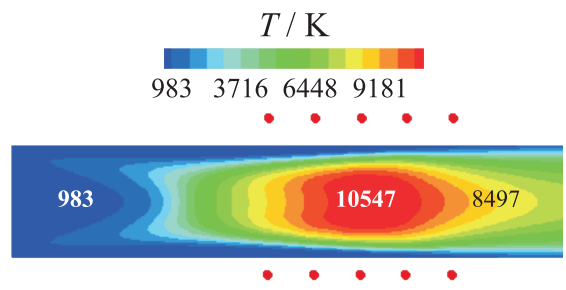

(b)

Figure 3 The translational temperature contours for thermally equilibrium $(a)$ and nonequilibrium $(b)$ gas-phase models: air, regime \# $1(P=2000 \mathrm{~Pa})$. Refer Sakharov et al., p. 371.)

subsonic flows over cylindrical model with $D=140 \mathrm{~mm}$. Figure 2 demonstrates the pressure counter and streamlines in the test chamber, around the model, and in discharge channel for regime \# 1 .

The calculation results using thermally nonequilibrium gas-phase model are presented on Fig. 3 for regime \# 1. The discrepancies in translational temperature maxima in discharge channel for thermally equilibrium $(a)$ and nonequilibrium $(b)$ gas phase models were about $3 \%$. The vibrational and electron temperatures obtained in the framework of all multitemperature models used are essentially differed from the translational ones in the torch. 


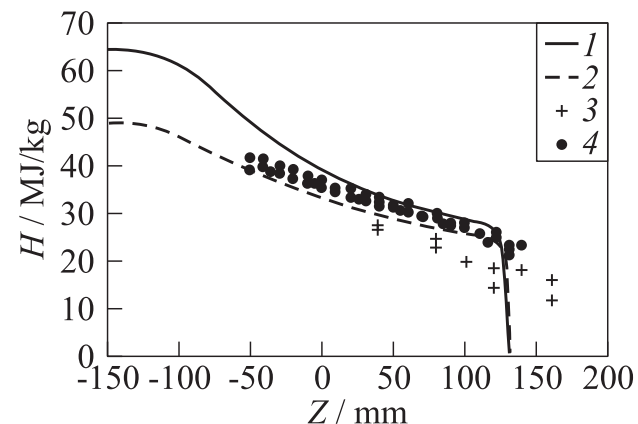

(a)

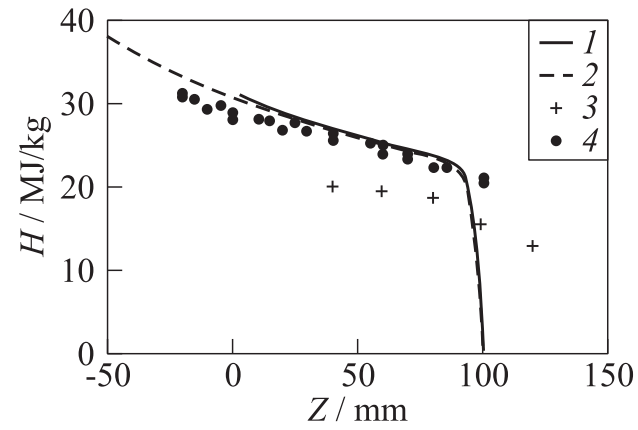

(b)

Figure 4 Enthalpy evolution along air plasma flow axis for regime \#2 $(P$ $=3700 \mathrm{~Pa})(a)$ and regime \#3 $(P=7800 \mathrm{~Pa})(b): 1$ and $2-$ numerical data of IPM and IM MSU, respectively; and 3 and 4 - measurements using enthalphymeters with the Euromodel geometry and with hemispherical nose, respectively

Further downstream, all temperatures become close to each other. The flow distributions for IPM and IM MSU approaches are nearer to each other for regime \# 3 in comparison with regimes \# 1 and \# 2. It means that IM MSU chemically nonequilibrium approach leads to IPM chemically equilibrium ones for higher pressure. But it is necessary to note that the chemically nonequilibrium model is better for using for low pressure. Some distinctions between IPM and IM MSU solutions can be observed in gas mixture composition.

The enthalpy evolution along the symmetric axis obtained by direct enthalpy measurements and by numerical simulation by IPM (obtained by S. Vasil'evskii) and IM MSU codes have been compared for two cases of subsonic air plasma flows and showed a good agreement as one can see in Fig. 4. Enthalpy measurements in high-enthalpy subsonic air flows using two different enthalpymeters with the Euromodel geometry (25-millimeter radius) and with hemispherical (15-millimeter radius) nose were performed. One can see that the direct enthalpy measure- 
ments held with the Euromodel probe were far from the results obtained by the hemispherical enthalpy probe and were quite different from the value obtained by both IPM and IM MSU numerical tools.

Once again, with higher pressure, the coincidence of the numerical results are better for both approaches since operating conditions are nearer to the equilibrium assumptions made by IPM.

\subsection{Stagnation Point Simulation for Air Flow}

To provide calculation by IM MSU approach heat flux envelopes (abacuses), classic IPM boundary layer gamma code [6] was modified for calculation thermally equilibrium and thermally nonequilibrium BL. Values for BL calculations (positions of BL outer edge, the sets of dimensionless parameters, etc.) were taken from the results of chemically nonequilibrium and thermally equilibrium (or not) N-S solutions for cold cupreous surface.

The numerical calculations for thermally nonequilibrium air flow were performed for the lowest pressures (regimes \#1 and \#2). The comparisons of solutions obtained by $\mathrm{N}-\mathrm{S}$ and $\mathrm{BL}$ codes have shown good resolution of BL by $\mathrm{N}-\mathrm{S}$ code. The heat maps obtained were in accord with $\mathrm{N}-\mathrm{S}$ calculations (Figs. 5 and 6).

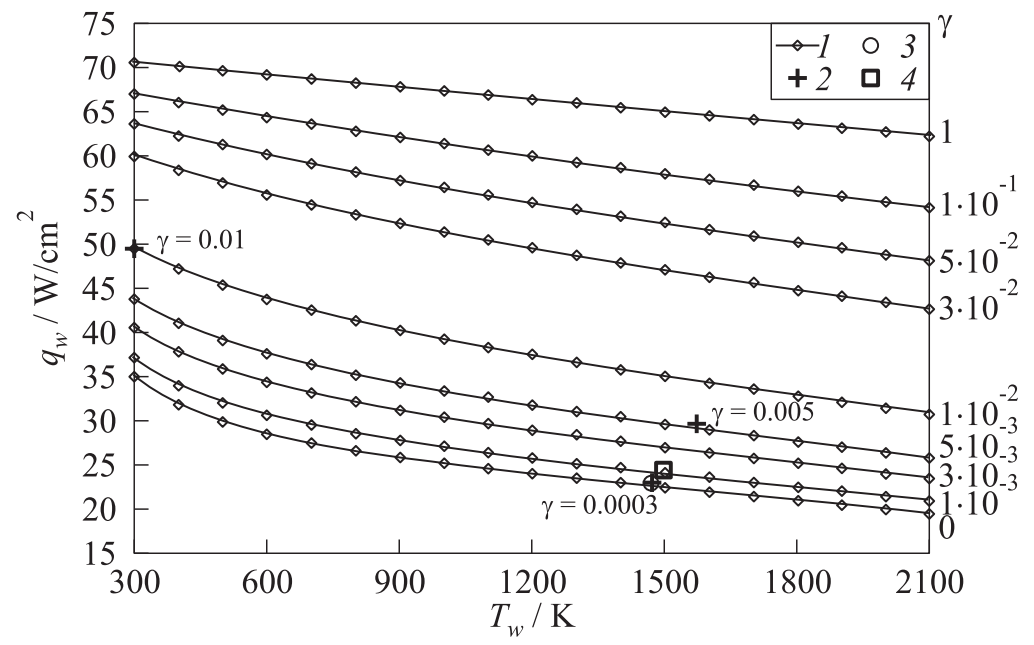

Figure 5 Heat fluxes to $D=140 \mathrm{~mm}$ flat-face model in IPG-4 air plasma, regime \# 1: 1 - results by BL code; 2 - results by $\mathrm{N}-\mathrm{S}$ code; 3 - experiment (tile); and 4 experiment $(\mathrm{SiC})$ 


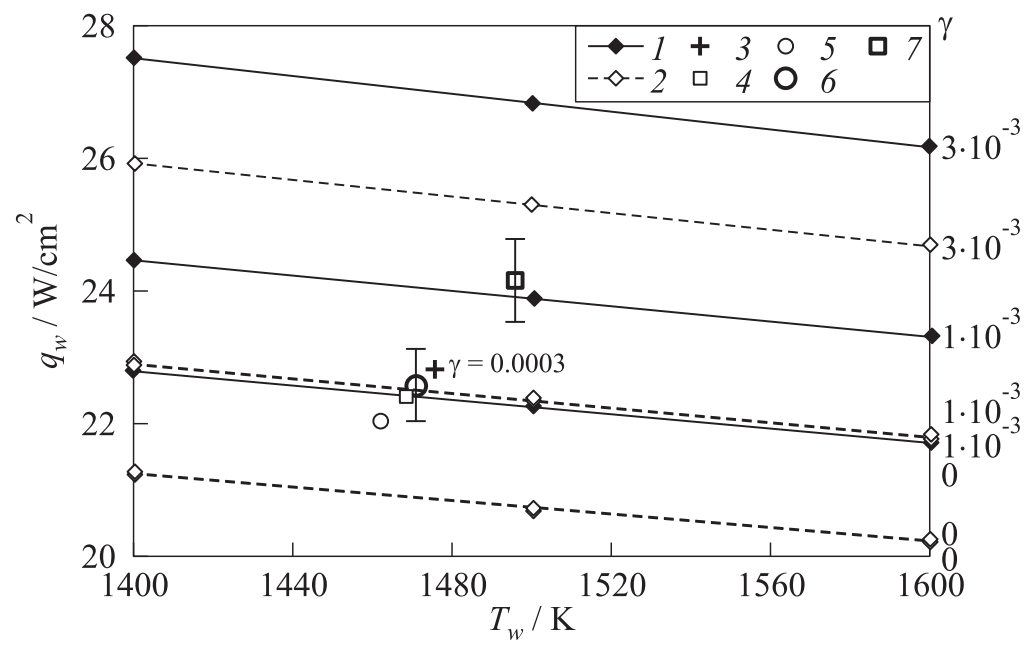

Figure 6 Heat fluxes $D=140 \mathrm{~mm}$ flat-face model in IPG-4 air plasma, regime \# 1 : 1 - the results by TEQ BL code; $3\left(\gamma=3 \cdot 10^{-4}\right), 6(\mathrm{SiC})$, and 7 (tile) - the results by TEQ N-S code; 2 - the results by TNEQ BL code; $4\left(T_{w}^{\mathrm{el}}=T_{w}^{v}=T_{w}\right)$ and 5 $\left(q_{w_{n}}^{v}=q_{w_{n}}^{\mathrm{el}}=0\right)$ - the results by TNEQ N-S code

Calculated heat flux abacuses have been compared with the same ones obtained by original IPM Gamma code (OGC) and modified IPM Gamma code (MGC) (necessary data were taken from IM MSU results of nonequilibrium N-S solutions). The abacuses calculated by IM MSU BL code and IPM (MGC) were in a good agreement for regimes \# 1 but distinctions were larger for regimes \# 2 and \# 3. It is necessary to note that in the IPM MGC, the flow parameters and values of species mass concentrations $C_{i e}$ were taken from IM MSU results of nonequilibrium $\mathrm{N}-\mathrm{S}$ solutions but positions of BL outer edge, flow vorticity, and the sets of some dimensionless parameters were taken from IPM calculations of LTE N-S equations. But the main reason of discrepancies observed between IPM and IM MSU approaches can be explained by different chemical model used.

The experimental value for the $\mathrm{SiC}$ sample and for the silica-based material which constitute the holder of the sample tested in plasma flows are reported on the graph by the squares and circles, respectively.

The heat flux maps for thermally nonequilibrium airflow is shown in Fig. 6 for regime \#1. Symbols TEQ and TNEQ in the caption of Fig. 6 refer to thermally equilibrium and nonequilibrium gas phase models, correspondingly. Blank rhombs correspond to thermally equilibrium and nonequilibrium BL for vibrational and electron temperatures. The thermally nonequilibrium model took effect on heat fluxes in the range of several percent for IPG-4 test conditions. 
Table 3 Effective recombination probability for air Pre-X conditions

\begin{tabular}{ccccc}
\hline Regime IPG-4, air & $P_{0}, \mathrm{~Pa}$ & $\begin{array}{c}T_{\mathrm{re}}, \mathrm{K} \\
(\varepsilon=0.85)\end{array}$ & $\gamma_{w}$ & IPM $\left(\gamma_{\mathrm{ref}}=1\right)$ \\
\hline 1 & 2000 & 1500 & 0.002 & 0.006 \\
2 & 3800 & 1540 & 0.0006 & 0.0043 \\
3 & 7800 & 1580 & 0.00001 & 0.0029 \\
\hline
\end{tabular}

In spite of some difference in abacus as a whole, calculated by IPM and IM MSU, the corresponding values of $\gamma$ (Table 3 ) obtained for low-catalytic materials are of the same order of magnitude or so that are characteristic of a low catalytic material (as expected for $\mathrm{SiC}$ samples). This duplication predicts the reduction of the wall temperature of the $\mathrm{SiC}$ sample on 300-350 K with respect to fully catalytic wall at the peak heating part of the trajectory.

\section{MARS SAMPLE RETURN ORBITER DUPLICATED $\mathrm{CO}_{2}$ CONDITIONS}

Following the same scheme as for the air case, ONERA provided trajectory points corresponding to the MSRO case to be duplicated with the ground test facilities. The application of LHTS theory to this case showed the difficulty to obtain a fully duplication of the free flight conditions due to the size of the nose radius of the MSRO. A partial duplication has been engaged which does not take into account the velocity gradient near the surface.

In Table 4, the operating conditions for the MSRO consideration are summarized. The point \#1 is impossible to reach with the plasmatron due to too low pressure; however, as previously done for air, a shift of pressure was applied to the point \# 1 and, for duplication purposes, a test at $2000 \mathrm{~Pa}$ was produced.

The $\mathrm{SiC}$ sample installed in the full-scale silica-based thermal protection tile has been tested in the high-enthalpy subsonic $\mathrm{CO}_{2}$ flows (Fig. 7) under conditions of the three selected regimes (see below).

Table 4 The MSRO computed data for fully catalytic conditions

\begin{tabular}{ccccc}
\hline Trajectory point, $\mathrm{CO}_{2}$ & $\begin{array}{c}\text { Flux, } \\
\mathrm{kW} / \mathrm{m}^{2}\end{array}$ & $\begin{array}{c}T_{\text {re }}, \\
\mathrm{K}\end{array}$ & $\begin{array}{c}\text { Stagnation pressure, } \\
\text { Pa }\end{array}$ & $\begin{array}{c}Z, \\
\mathrm{~km}\end{array}$ \\
\hline 1 & 146 & 1500 & 1049 & 55 \\
2 & 457 & 1500 & 3710 & 46 \\
3 & $189-838$ & 1500 & 9595 & 37 \\
\hline
\end{tabular}




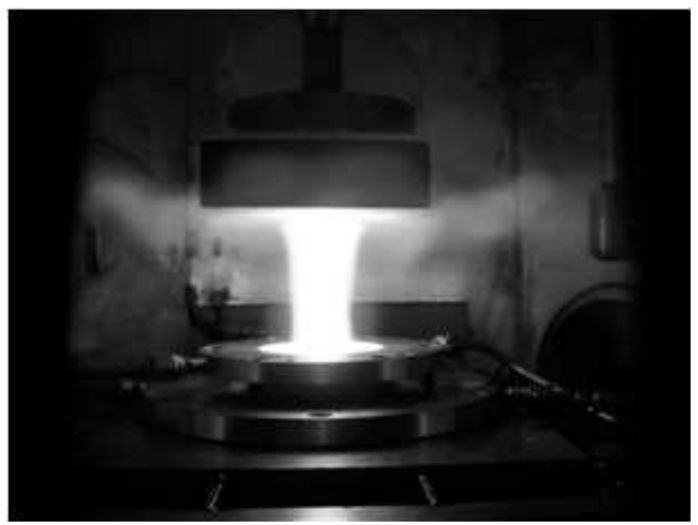

Figure 7 Full-scale silica-based tile with installed $\mathrm{SiC}$ sample in subsonic highenthalpy $\mathrm{CO}_{2}$ flow

Table 5 Parameters of the testing regimes

\begin{tabular}{ccccc}
\hline Regime IPG-4, $\mathrm{CO}_{2}$ & $N_{\mathrm{ap}}, \mathrm{kW}$ & Efficiency & $P_{\mathrm{ch}}, \mathrm{Pa}$ & $Z_{\text {exit }}, \mathrm{mm}$ \\
\hline 1 & 47.2 & 0.35 & 2000 & 130 \\
2 & 51.5 & 0.375 & 3700 & 130 \\
3 & 47.7 & 0.415 & 9600 & 100 \\
\hline
\end{tabular}

Table 6 Measured temperatures of the $\mathrm{SiC}$ and silica-based samples in subsonic highenthalpy $\mathrm{CO}_{2}$ flows

\begin{tabular}{ccc}
\hline Regime IPG-4, & \multicolumn{2}{c}{$T_{\mathrm{re}}, \mathrm{K}(\varepsilon=0.85)$} \\
\cline { 2 - 3 } $\mathrm{CO}_{2}$ & Silica & $\mathrm{SiC}$ \\
\hline 1 & 1350 & 1393 \\
2 & 1450 & 1426 \\
3 & 1480 & 1536 \\
\hline
\end{tabular}

Also, the silica-based thermal protection tile has been tested in the same three regimes for comparison.

Parameters of the testing regimes and measured temperatures of the $\mathrm{SiC}$ samples and silica-based tile at the center are shown in Tables 5 and 6 .

\section{1 $\quad \mathrm{CO}_{2}$ Plasma Flow Simulation}

The calculations using thermally equilibrium and thermally nonequilibrium gasphase model have been carried out for IPG-4 $\mathrm{CO}_{2}$ test regimes: \# 1 and \# 2 . 


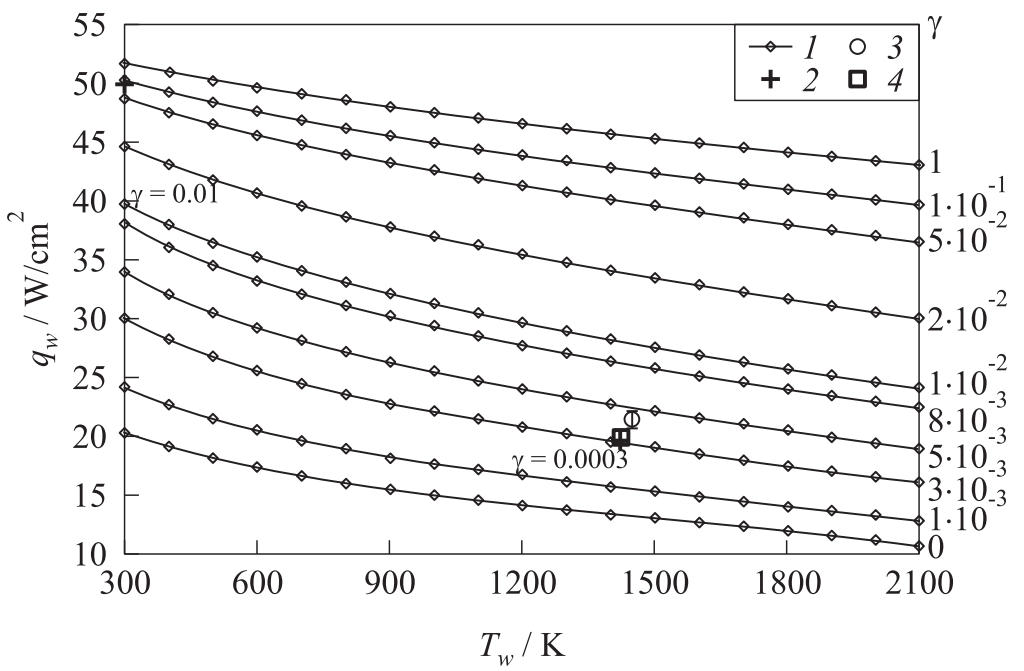

Figure 8 Heat fluxes to $D=140 \mathrm{~mm}$ flat-face model in IPG-4 $\mathrm{CO}_{2}$ plasma, regime \# 1: 1 - results by $\mathrm{BL}$ code; 2 - results by $\mathrm{N}-\mathrm{S}$ code; 3 - experiment (tile); and 4 - experiment ( $\mathrm{SiC}$ )

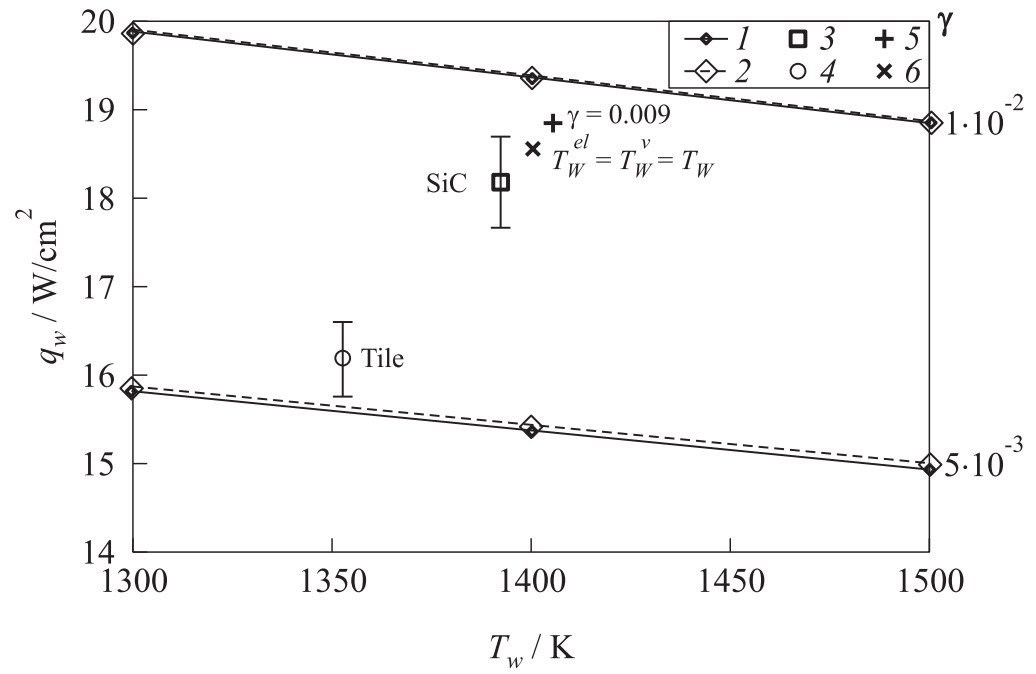

Figure 9 Heat fluxes to flat-face model $D=140 \mathrm{~mm}$ in IPG- $4 \mathrm{CO}_{2}$ plasma, regime \# 1: 1 - the results by TEQ BL code; 2 - the results by TNEQ BL code; 3 (experiment, $\mathrm{SiC}$ ); 4 (experiment, tile); and 5 (calculation, $\gamma=0.009$ ) - the results by TEQ $\mathrm{N}-\mathrm{S}$ code; $6\left(T_{w}^{\mathrm{el}}=T_{w}^{v}=T_{w}\right)-$ the results by TNEQ N-S code 
Unfortunately, it has not been got the stable numerical solution for regime \# 3 . It could be explained by inadequacy between quasi-3D statement of problem and real-3D ICP flow in plasmatron for these parameters.

The calculations using thermally nonequilibrium gas-phase model have been carried out for regime \# 1.

The discrepancies in solutions for thermally equilibrium and thermally nonequilibrium gas phase models were considerably less compared with airflow regimes. The vibrational and electron temperatures in the discharge channel obtained in the framework of all multitemperature models are differed less in comparison with air flows.

\subsection{Stagnation Point Simulation for $\mathrm{CO}_{2}$ Flow}

The heat flux abacuses were calculated by IM MSU chemically nonequilibrium BL codes for regimes \# 1 (Fig. 8) and \# 2 in $\mathrm{CO}_{2}$ flows. Using of thermally non-equilibrium model took effect on heat fluxes in the range of several percent (Fig. 9).

The calculated of the stagnation point temperature to $\mathrm{SiC}$ samples and calculated abacuses have allowed to determine the catalytic properties $\mathrm{SiC}$ material for $\mathrm{CO}_{2}$ flows (Table 7).

Table 7 Effective recombination probability for $\mathrm{CO}_{2}$ MSRO conditions

\begin{tabular}{ccccc}
\hline Regime, $\mathrm{CO}_{2}$ & $P_{\mathrm{ch}}, \mathrm{Pa}$ & $\begin{array}{c}T_{\mathrm{re}}, \mathrm{K} \\
(\varepsilon=0.85)\end{array}$ & $\gamma_{w}$ & $\mathrm{IPM}\left(\gamma_{\mathrm{ref}}=1\right)$ \\
\hline 1 & 2000 & 1390 & 0.008 & 0.0094 \\
2 & 3700 & 1430 & 0.003 & 0.0066 \\
3 & 9600 & 1540 & - & 0.0049 \\
\hline
\end{tabular}

\section{CONCLUDING REMARKS}

1. Subsonic air and $\mathrm{CO}_{2}$ jets and heat transfer under the IPG-4 plasmatron test conditions were studied by CFD modeling.

2. Navier-Stokes code using thermochemical models of various complexity was approved for modeling IPG-4 subsonic flow.

3. The calculated at the stagnation point temperatures to $\mathrm{SiC}$ samples and comparison with experimental data in air and $\mathrm{CO}_{2}$ flow allowed to define the catalytic property of $\mathrm{SiC}$. 
4. The calculated at the stagnation point heat fluxes to $\mathrm{SiC}$ samples do not depend on thermal nonequilibrium, but strongly depend on chemical model used.

5. The results obtained so far are the same in order of magnitude and show that the samples are a low catalytic material in regards with the specified operating conditions of both Pre-X and MSRO vehicles.

6. The duplication predicts the reduction of the wall temperature of the $\mathrm{SiC}$ sample on $300-350 \mathrm{~K}$ with respect to fully catalytic wall at the peak heating part of the trajectories

\section{ACKNOWLEDGMENTS}

This work has been supported by the INTAS/CNES No. 53-5117 and Russian Foundation for Basic Research No. 08-01-00447 projects.

\section{REFERENCES}

1. Verant, J.-L., N. Perron, O. Gerasimova, M. Balat-Pichelin, A. Kolesnikov, V. Sakharov, and P. Omaly. 2006. Microscopic and macroscopic analysis for TPS $\mathrm{SiC}$ material under Earth and Mars re-entry conditions. 14th AIAA/AHI International Space Planes, Hypersonic Systems and Technologies Conference Proceedings. Canberra, Australia. 6-9 November. 25 p. www.aiaa.org.

2. Kolesnikov, A. 1993. Conditions of simulation of stagnation point heat transfer from a high enthalpy flow. Fluid Dynamics 28:131-37.

3. Kolesnikov, A. 1994. The aerothermodynamic simulation in sub- and supersonic high enthalpy jets: Experiments and theory. 2nd European Symposium on Aerothermodynamics for Space Vehicles Proceedings. Noordwijk, The Netherlands. ESA SP-367. P. 583-90.

4. Kolesnikov, A. F. 2000. The concept of local simulation for stagnation point heat transfer in hypersonic flows: Applications and validation. AIAA Paper No. 20002515 .

5. Kolesnikov, A.F., and L. Marraffa. 1999. An analysis of stagnation point thermochemical simulation by plasmatron for Mars probe. AIAA Paper No. 99-3564.

6. Vasil'evskii, S.A., and A.F. Kolesnikov. 2000. Numerical simulation of equilibrium induction plasma flows in a cylindrical plasmatron channel. Fluid Dynamics 35(5):769-77.

7. Sakharov, V.I., and V. G. Gromov. 2005. CFD modeling of thermally and chemically nonequilibrium flows in discharge channel and in under-expanded plasmatron jet over a butt-end probe. 5th European Symposium on Aerothermodynamics for Space Vehicles Proceedings. Cologne, Germany. SP 563. P. 119-23. 
8. Sakharov, V. I., V. G. Gromov, A.F. Kolesnikov, and A. N. Gordeev. 2007. CFD modeling of thermally and chemically nonequilibrium air flows in discharge channel and in under-expanded plasmatron jets over a butt-end probe. 2nd European Conference for Aerospace Sciences (EUCASS). Brussels. CD-ROM.

9. Sakharov, V.I. 2007. Numerical simulation of thermally and chemically nonequilibrium flows and heat transfer in underexpanded induction plasmatron jets. Fluid Dynamics 42(6):1007-16.

10. Kolesnikov A. F. 2000. Stefan-Maxwell relations for multicomponent ambipolar diffusion and thermal-baro-diffusion effects in two temperature plasmas. AIAA Paper No. 2000-2570.

11. Afonina, N.E., and V.G. Gromov. 1998. Thermochemical nonequilibrium computations for a MARS express probe. 3rd European Symposium on Aerothermodynamics for Space Vehicles Proceedings. ESTEC, Noordwijk, The Netherlands. P. $179-86$.

12. Reid, R. C., J. M. Prausnitz, and T.K. Sherwood. 1977. The properties of gases and liquids. New York: McGraw-Hill Book Company.

13. Afonina, N. E., V. G. Gromov, and V. I. Sakharov. 2005. HIGHTEMP technique for high temperature gas flows simulations. 5th European Symposium on Aerothermodynamics for Space Vehicles Proceedings. Cologne, Germany. SP 563. P. 323-28. 